This is a post-print of a paper of mine which appeared in Erkenntnis, vol. 76(1), 2012, pp. 73-89. It is not meant to replace the original, which can be retrieved at http://www.springerlink.com/content/t7v77784547n2212/.

\title{
Scientific Representations as Limiting Cases
}

\author{
Steffen Ducheyne
}

\begin{abstract}
In this essay, I shall show first of all that the socalled inferential (Suárez 2003 and 2004) and interpretational (Contessa 2007) accounts of scientific representation are respectively unsatisfactory and too weak to account for scientific representation (pars destruens). Along the way, I shall also argue that the pragmatic similarity (Giere 2004 and 2010) and the partial isomorphism (da Costa and French 2003 and French 2003) accounts are unable to single out scientific representation. In the pars construens I spell out a limiting case account which has explanatory surplus vis à vis the approaches which I have previously reviewed. My account offers an adequate treatment of scientific representation, or so I shall try to argue. Central to my account is the notion of a pragmatic limiting case, which will be characterized in due course.
\end{abstract}

\section{Introduction}

Scientific representation is a many-headed beast which involves a game of "selective resemblance and non-resemblance" (van Fraassen 2008, p. 14). In recent years there has been a growing literature which endeavours to address the constitution problem of scientific representation (for instance, Callender and Craig 2006, Contessa 2007, French 2003, Frigg 2006, Giere 2004 and 2010, Hughes 1997, Suárez 2003 and 2004). In this paper, I pave the way for a new proposal: the limiting case account of scientific 
representation, which will be spelled out in section 5. I will focus on the constitution question of scientific representation: in virtue of what does a model scientifically represent a part of the empirical world? In shall begin this essay by questioning the tenability of Callender and Craig's view that there is no special problem of scientific representation in section 2. In section 3 I argue that Suárez' deflationary proposal cannot provide a satisfactory answer to the issue at hand and, in section 4, I argue that Contessa's interpretational account is incomplete as it stands.

\section{Griceanism and its Problems}

Some have argued that there is no such thing as the problem of scientific representation. Craig Callender and Jonathan Cohen have recently suggested that if we adopt a General Gricean approach to representation, "we won't need separate theories to account for artistic, linguistic, representation, and culinary representation; instead the General Gricean proposes that all these types of representation can be explained (in a unified way) as deriving from some more fundamental sorts of representations, which are typically taken to be mental states," a treatment which they deem "economical and natural" (Callender and Cohen 2006, p. 70, cf. p. 75). Their general explanatory strategy encompasses the view that "virtually anything can be stipulated to be a representational vehicle for the representation of virtually anything" - a view to which Steven French has objected by pointing out that not everything can serve as a scientific model of a physical system, for "if the appropriate relationships are not in place between the relevant properties then the "model" will not be deemed scientific" (French 2003, p. 1478) - although "there are plausibly pragmatic constraints on which representational vehicles and targets are used in particular cases" (Callender and Cohen 2006, p. 74). To objections à la French they respond: "We propose that intuitions to the effect that such and such cannot serve as a model are best understood as reflecting the unlikelihood of anyone's using such 
and such as a model, given certain assumptions about pragmatic purposes." (ibid., p. 76, footnote 6 [italics added]). While all representational vehicles have the capacity of serving as fullblooded representational vehicles in principle, they may not do so in practice because they fail to serve the purposes at hand (ibid., pp. 74-75). Just as in non-scientific representation, the questions about the utility of representational vehicles are questions about the pragmatics of things that are representational vehicles, not questions about their representational status per se (ibid., p. 75). Hence: "while there may be outstanding issues about representation, there is no special problem about scientific representation" (ibid., p. 77, cf. p. 83). Exit the problem of scientific representation?

Does it follow from Callender and Cohen's premise, that the capacity of all kinds of representation (linguistic, artistic, scientific, etc.) are constrained by pragmatic considerations, that there is no problem of scientific representation at all? Callender and Cohen's far-reaching conclusion seems to follow only if they are able to show that the pragmatic elements constraining both artistic as well as scientific representation are not fundamentally different from each other. In other words, what they fail to show is that there are no relevant differences qua domain-specific pragmatics in artistic and scientific representation. Artists are in numerous cases constrained in their use of materials of choice of colours by the emotions or thought processes they seek to elicit in their audiences. The painting The Third of May (1814) would not have its dramatic effect had Francisco de Goya used vivid colours and a bright background to represent the horrors of Napoleon's soldiers against the Spanish in 1808. On the other hand, scientists are constrained in their choice of models by the kinds of descriptions or explanations of particular natural phenomena they seek to provide. ${ }^{1}$ For instance, when studying a continuous phenomenon, a non-continuous function will not be first choice. It is true that artists' and scientists'

${ }^{1}$ All I need to assume here is that in scientific representation providing descriptions or explanations of natural phenomena is the dominant pragmatic constraint. 
choices for a specific representational vehicle are constrained by pragmatic considerations, but they are constrained by pragmatic considerations of a different kind. Unless Callender and Cohen show that such differences qua pragmatic elements are not relevant, their conclusion that there is no problem of scientific representation does not follow. Moreover, their general and economical framework, according to which "the varied representational vehicles used in scientific settings (models, equations, toothpick constructions, drawings, etc.) represent their targets (the behaviour of ideal gases, quantum state evolution, bridges), by virtue of the mental states of their makers/users" (ibid., p. 75 [italics added]), comes at a high price in terms of explanatory power - in my opinion, at a price too high. An account of scientific representation needs to explain in more detail how it is that we can draw inferences from representations in science, to come up with a more elaborate picture about the pragmatics of scientific representation, and to show how scientific representations can be seen as distinct from non-scientific representations. Callender and Cohen's account remains silent on these matters and nothing particularly useful follows from it in terms of coming to terms with concrete scientific representations. Accepting Callender and Cohen's proposal means giving up on scientific representation all too soon. ${ }^{2}$

\section{Deflationism and its Problems}

Mauricio Suárez has defended a position which is less radical than Callender and Cohen's Gricean account: deflationism. Characteristic of his account is the refusal to spell out what counts as realizing the "surface features" of scientific representation. In their early work on scientific representation, both Ronald N. Giere and Bas C. van Fraassen embraced standard similarity and isomorphism (see Giere 1988, p. 80, Giere 1999 and van Fraassen 1980 , p. 45, p. 64). In two recent thought-provoking papers, Suárez has tried to debunk similarity [sim], according to which $A$

\footnotetext{
${ }^{2}$ In section 5 I shall provide additional support of the claims made here.
} 
represents $B$ if and only if $A$ is similar to $B$, and isomorphism [iso], according to which $A$ represents $B$ if and only if the structure exemplified by $A$ is isomorphic to the structure exhibiting by $B$, as adequate explications of scientific representation, by arguing that [sim] and [iso] do not qualify as the constituents of scientific representation, which he defines as follows, "the relation $\mathrm{R}$ between $\mathrm{A}$ and $\mathrm{B}$ are the constituents of representation of $\mathrm{B}$ by $\mathrm{A}$ if and only if R's obtaining is necessary and sufficient for A to represent B" (Suárez 2003, p. 230). Suárez' criticism is directed at "theories that attempt to reduce scientific representation to similarity or isomorphism" (Suárez 2003, p. 225 [italics added]). Moreover, "[t]hese reductive theories aim to radically naturalize the notion of representation, since they treat scientists' purposes and intentions as non-essential to representation" (ibid. [italics added]). ${ }^{3}$ According to Suárez, $[\mathrm{sim}]$ and [iso] are facts about the source and target objects (and their properties), not about the essentially intentional judgements of representation-users scientific representation is triadic instead of dyadic. Scientific representation is a not an object-object relation but rather a relation between objects and the internal states of their users.

A substantive account of scientific representation "provides us with necessary and sufficient conditions for a source to represent its target" (Suárez 2003, p. 226). Suárez proposal furthermore encompasses the view that we should take a "deflationary" or "minimist" attitude towards scientific representation (Suárez 2004, pp. 770-771), which basically entails two things: (1) we should abandon the quest for a substantive theory because representation is not "the kind of thing that requires, or admits" such theory and (2) we can at best aim to describe its "surface features" of representations and should stop seeking for "deeper features to representation" (ibid., p. 771). According to Suárez' inferential account, the two surface features are: the representational force of a source and the capacity of surrogate reasoning, i.e. the capacity of

${ }^{3}$ In this context, Suárez refers to Giere 1988 and 1999 and van Fraassen 1980 as defences of [sim] and [iso], respectively (Suárez 2003, p. 227). 
drawing inferences about the target from the model. Correspondingly, he defines representation as:

[inf]: $A$ represents $B$ if only (i) the representational force of $A$ points toward $B$, and (ii) $A$ allows competent and informed agents to draw specific inferences regarding $B$. (Suárez 2004, p. 773)

(i) and (ii) are obviously necessary conditions. ${ }^{4}$ The type and level of competence and information required in the surrogate reasoning process, which may be deductive, inductive, analogical, etc., is a pragmatic skill that depends on the aim and the context of the inquiry. In a sense, all has been said about representation in science.

Suárez makes it sound as if unravelling only necessary conditions for scientific representation is what endorsing a deflationary stance amounts to. However, the difference between endorsing a deflationary versus a substantive stance does not consist in the addition or subtraction of sufficient conditions. What makes an account deflationary is not the absence of the sufficient conditions, but rather the refusal to further spell out what counts as realizing the representational force and the capacity for surrogate reasoning. Rather than being an account of scientific representation, [inf] is thus the general form of an account of representation: it is the blank to be filled in every instance of representation. Deflationism amounts to the view that a theory of representation should not fill in this blank.

The problem is, however, that Suárez provides no real argument for the claim that a substantive account of scientific representation is impossible in principle (cf. Contessa 2007, pp. 4950). Suárez furthermore explicates representation in terms of representational force and the capacity for surrogate reasoning. Now, unless an detailed account of representational force is provided, which is currently lacking in Suárez' account, nothing much has been gained. Up until the present, he has simply replaced

${ }^{4}$ Suárez notes that he has "little to say about what makes one representation more accurate than another" (Suárez 2003, p. 226). Suárez explicitly deals with the constitution question of scientific representation and not with the normative question of scientific representation. 
ignotum per ignotum. Moreover, what we expect from an account of scientific representation is not so much the mere statement that we can make inferences, but rather an explanation of why and how it is that we can make such inferences.

Suárez furthermore offers five arguments against [sim] and [iso] (Suárez 2003, pp. 230-237):

1. The argument of variety: $[\operatorname{sim}]$ and [iso] do not apply to all representational devises.

2. The logical argument: $[$ sim] and [iso] do not possess the logical properties of representation. ${ }^{5}$

3. The argument from misrepresentation: $[$ sim $]$ and $[i s o]$ do not make room for the ubiquitous phenomena of mistargeting and/or inaccuracy.

4. The non-necessity argument: $[$ sim $]$ and $[i s o]$ are not necessary for representation - the relation of representation may obtain even if $[\operatorname{sim}]$ or $[i s o]$ fail.

5. The non-sufficiency argument: $[\mathrm{sim}]$ and $[i s o]$ are not sufficient for representation - the relation of representation may fail to obtain even if [sim], or [iso] hold.

However, his counterarguments only establish that naive [sim] and [iso] aren't necessary and sufficient conditions for scientific representation. According to Suárez, the defenders of the $[\mathrm{sim}]$ or [iso] account endorse the view that $A$ represents $B$, if and only if, $A$ is similar to $B$ or, if and only if, the structure exemplified by $A$ is isomorphic to the structure exhibiting by $B$, respectively. ${ }^{6}$ I shall label this similarity/isomorphism account naive similarity/isomorphism, because by definition it does not refer to the pragmatic aspects of scientific representation. Naive [sim] and [iso] clearly are untenable. For instance: in certain contexts, a user may consider a deck of cards to be similar or isomorphic to a pack of cigarettes. But nobody would contend that by itself the pack of cigarettes represents the deck of cards. If relevant interpretations by users are added, a deck of cards can represent a pack of cigarettes as is clear from the following example. Suppose, for instance, that

\footnotetext{
${ }^{5}$ This argument against [iso] and [sim] is taken over in Frigg 2006.

${ }^{6}$ I consider [iso], similarity qua form, as a specific version of [sim].
} 
an individual seeks to estimate roughly the volume needed to stash 3,000 packs of cigarettes but has no pack of cigarettes at hand. Suppose further that this individual does have a deck of cards in one of his pockets. He or she could estimate the required volume by calculating the volume needed to store 3,000 decks of cards, which (roughly) represent the volume of an identical number of packs of cigarettes. By adding certain pragmatic elements, the volume of the decks of cards represents the volume of the packs of cigarettes. This type of similarity I consider as pragmatic similarity/isomorphism, since it refers to the choice of one particular similarity/isomorphism relation to do the representing. In his later work on the matter, van Fraassen has emphasized the pragmatic dimension of representation and, more specifically he has argued that there are four characteristics involved in representation: (1) intentionality, which presupposes specific "reading conventions," (2) selectivity of aspects, (3) accuracy, which admits several degrees of fitting, and (4) context-relativity, which is entailed by the preceding characteristics) (van Fraassen 1994, p. 171; cf. van Fraassen 2008, pp. 22-26, p. 76). Da Costa and French have also emphasized the pragmatic features of their partial isomorphism proposal (da Costa and French 2003, pp. 16-20, pp. 33-36) and, recently, Giere has also come to focus on the pragmatic dimensions of similarity (Giere 2004, p. 743). Accordingly, scientific representation is essentially triadic: a subject uses a model to represent a physical system for certain purposes (ibid.). Giere explicitly rejects standard similarity: he notes that a model and a physical system are not similar by themselves (ibid., p. 747; id. 2010, pp. 274-275). ${ }^{7}$

${ }^{7}$ Others have also endorsed pragmatic [sim] (Teller 2001 and BailorJones 2003). According to Teller, what counts as similarity will depend on the level of accuracy one requires: "if the aim is prediction or explanation of quantitative detail one will need to specify the interests of the model users in more detail" (Teller 2001, p. 401 [emphasis added]). Moreover, he notes that because of the context-sensitivity involved in scientific representation, no general account of relevant similarity is required: "No general account is needed precisely because it is the specifics of any case at hand which provide the basis for saying what counts as relevant similarity. In other words, the very facts 
As Suárez' logical argument is directed at proponents of naive $[\operatorname{sim}]$ and [iso], his criticism does not seem to affect the pragmatic [sim] or [iso] accounts. That being said, it does not follow that pragmatic [sim] or [iso] provide an adequate solution to the constitution problem of scientific representation (see 5.1).

\section{The Interpretational Proposal}

Gabriele Contessa's interpretational account differs from Suárez inferential account in that it seeks to provide a substantive account of scientific representation (Contessa 2007). Contessa begins by distinguishing between three different meanings of representation: denotation, epistemic representation and faithful representation (ibid., pp. 52-56). The logo of the London Underground and a map of the London Underground denote the London Underground network. Denotation may be a matter of convention. However, contrary to the logo of the London Underground, a map of the London Underground represents the underground network in a stronger sense: namely, a user can perform (valid but not necessarily sound) surrogate inferences from the map to the network. This is an instance of what Contessa calls an epistemic representation. A vehicle ${ }^{8}$ is an epistemic representation of a certain target for a certain user if and only if the user is able to perform valid (though not necessarily sound) surrogate inferences from the vehicle to the target (ibid., p. 48, pp. 52-53, p. 67). Faithful, i.e. valid and sound, representation occurs when a vehicle (model) allows surrogate inferences that are not only valid but also sound, i.e. true of its target. Soundness is a matter of degree: a vehicle (model) does not need to be a perfect replica of its target: it faithfully represents some aspects

which make this demand impossible to meet also show that the demand was misguided to begin with." (ibid.). Daniela Bailer-Jones also endorsed pragmatic [sim], for she stressed that the users of models should agree upon the function for which a model is intended, decide which allowances are made for the model not to fit the data or the laws of nature, and select the aspects of a phenomenon which a model represents (Bailer-Jones 2003, p. 72).

${ }^{8}$ Note that 'vehicle' and 'model' are used interchangeably by Contessa. 
of the target and misrepresents other aspects (ibid., pp. 55-56, p. 60). Contessa's aim is to provide an adequate account of epistemic representation - while leaving over an account of faithful representation for future research (ibid., pp. 67-68).

According to Contessa, a vehicle is an epistemic representation of a certain target for a certain user if and only if the user adopts an interpretation of the vehicle in terms of the target (ibid.). A user interprets a vehicle in terms of a target if he takes facts about the vehicle to stand for (putative) facts about the target (ibid.). The notion of interpretation is spelled out in terms of analytic interpretation: a user adopts an analytic interpretation of a vehicle in terms of a target, if and only if (ibid., p. 58):

1. The user takes the vehicle to denote the target,

2. The user takes every object in $\Omega^{V}$ [the non-empty set of relevant objects in the vehicle] to denote one and only one object in $\Omega^{T}$ [the non-empty set of relevant objects in the target] and every object in $\Omega^{T}$ to be denoted by one and only one object in $\Omega^{V}$,

3 . The user takes every $n$-ary relation in $P^{V}$ [the possibly empty set of relevant properties and relations among objects in the vehicle] to denote one and only one relevant $n$-ary in $P^{T}$ [the possibly empty set of relevant properties and relations among objects in the target] and every $n$-ary relation in $P^{T}$ to be denoted by one and only one $n$-ary relation in $P^{V}$.

4. They take every $n$-ary function in $\Phi^{V}$ [the set of relevant functions from $\left(\Omega^{V}\right)^{n}$ to $\Omega^{V}$ ] to denote one and only one relevant $n$-ary function in $\Phi^{T}$ [the set of relevant functions from $\left(\Omega^{T}\right)^{n}$ to $\Omega^{T}$ ] and every $n$-ary function in $\Phi^{T}$ to be denoted by one and only one $n$-ary relation in $\Phi^{V}$.

Contessa admits that not all but most scientific representations are analytic interpretations and adds that focussing on analytic representation will "simplify the discussion without any loss of generality" (ibid., p. 58). Contessa's account clearly does not require that every object in the model denotes some object in the corresponding system or that the objects in the system actually have all the properties instantiated by the objects that stand for them in the model (ibid., p. 59). A user's background knowledge will allow "to assess which properties of objects of the model are idealizations 
or approximations and would lead to unsound inferences about the properties of the corresponding objects in the system" (ibid., p. 60).

In comparison to Suárez' approach on the subject, Contessa's proposal is certainly appealing since it is more explanatory of scientific representation. Contessa notes: "On the inferential conception, the user's ability to perform inferences from a vehicle to a target seems to be a brute fact, which has no deeper explanation. [...] On the interpretational conception, on the other hand, a user's ability to perform pieces of surrogate reasoning not only is not a mysterious skill but it is an activity that is deeply connected to the fact that the vehicle is an epistemic representation of the target for that user." (ibid., p. 61). In other words, (analytic) interpretation is not just a surface feature or symptom of representation: one performs surrogate inferences in virtue of the fact that the user interprets the vehicle in terms of the target.

However, Contessa's account of epistemic representation or analytic interpretation is at best incomplete as it stands. According to Contessa's substantial account, scientific representation basically occurs if and only if: (1) a user takes the model as a whole to stand for a target, (2) a user takes some of the components of the model to stand for some of the components of the target, and (3) a user takes some of the properties of and relations among the objects in the model to stand for some of the properties of and relations among the objects of the target (ibid., p. 59). Note that an answer to the question in virtue of what does $\Omega^{V} / P^{V}$ denote $\Omega^{T} / P^{T}$ is not provided. The problem with Contessa's proposal is that the notion of denotation, on which his account is based, is too weak to characterize scientific representation, as is shown by the following example. The example below meets Contessa's requirements for analytic interpretation, but it does not seem to qualify as a scientific representation. Suppose that seven runners have just competed in a contest. After the finish we take a group picture with all runners standing next to each other in a random fashion. Next, above each of the runners on the photograph we add identical circles coloured with one of the seven primary colours. Additionally, we denote the runner who had the best time in the contest by the colour with the 
highest wavelength, the runner who had the second best time by the colour with the second highest wavelength, and so on for all other runners. On the basis of this interpretation, we can infer, for instance, that the individual on the photograph with a green circle above finished fourth. This qualifies as an analytic interpretation since we take the colours - ordered in terms of their wavelengths (from highest to smallest) - to denote the order of who finished (from first to last). We take the relation having highest wavelength to stand for being the fastest runner, having the second highest wavelength for being the second fastest runner, and so on. However, this example does not seem to qualify as a straightforward scientific representation. Denotation alone, it seems, is too weak to capture scientific representation.

If the above line of reasoning is correct, then something is missing in Contessa's proposal. If it can be made plausible that vehicles scientifically represent their targets in virtue of a more fundamental relation between vehicles and their targets, which is exactly what I endeavour to do in the following section, then it is possible to advance upon Contessa's account and to arrive at a more adequate explication of scientific with an explanatory surplus.

\section{The Pragmatic Higher-level Limiting Case Account of Scientific Representation}

\subsection{Preliminaries and Motivation}

An account of scientific representation should keep the question in virtue of what does a scientific representation represent a certain system (i.e., the constitution problem of scientific representation) separate from the question in virtue of what does a representation represent a certain system accurately or truthfully (i.e. the normative problem of representation) (Suárez 2004, pp. 767-768). Correspondingly, I shall deal with both issues separately in subsection 5.2 and 5.3, respectively.

At the end of the previous section, I have argued that 
denotation by itself is too weak to explicate scientific representation. It seems therefore that a more fundamental relation between models $(M)$ and their targets $(T)$ is required to explain why users interpret scientific models analytically in the first place. Defenders of pragmatic similarity (Giere 2004 and 2010), partial isomorphism (da Costa and French 2003 and French 2003), and homomorphism (Mundy 1986) all claim to have found this more basic relation. I will try to argue that they are misguided in this assumption. $^{9}$

Let us begin with a quick discussion of the partial isomorphism account and the pragmatic similarity account, respectively. A partial structure, $A$, is defined as set-theoretic structure: $A=\left\langle D, R_{i}\right\rangle_{i \in I}$, where $D$ is the (non-empty) domain and each $R_{i}$ is a partial relation (da Costa and French 2003, pp. 16-20 and French 2003, p. 1480). Characteristic of a partial structure is that the relations are defined only for some elements of the domain. Each partial relation can be viewed as an ordered triple $<R_{1}, R_{2}$, $R_{3}$ >, where $R_{1}, R_{2}$, and $R_{3}$ are mutually disjoint sets such that $R_{1} \cup$ $R_{2} \cup R_{3}=A^{n}$ and such that $R_{1}$ is the set of $n$-tuples that belong to $R$, $R_{2}$ is the set of $n$-tuples that do not belong to $R$, and $R_{3}$ is the set of $n$-tuples for which it is not defined whether they belong to $R$ or not. ${ }^{10}$ Accordingly, partial isomorphism between two partial structures $A$ and $A^{\prime}$ obtains, if and only if, a partial substructure of $A$ is isomorphic to a partial substructure of $A^{\prime}$ (da Costa and French 2003, p. 49 and French 2003, p. 1480). According to da Costa and French, the above formal characterization captures an important feature of scientific representations, namely that they involve idealizations and approximations:

Both our everyday and scientific beliefs concern representations that are not determinate, not tight, not complete; they are idealizations and approximations, they are imperfect, and they are partial,

\footnotetext{
${ }^{9}$ As the arguments against (pragmatic) homomorphism are analogous to those against (pragmatic) similarity, I will omit further discussion of homomorphism in what follows.

${ }^{10}$ Note that in the limiting case, where $R_{3}$ is empty, a partial structure becomes a total structure.
} 
reflecting our partial knowledge and understanding of the world. (da Costa and French 2003, p. 17, cf. p. 19)

In this context, it is claimed that idealization, abstraction and approximation can be accommodated within the partial isomorphism account (French 2003, p. 1480). ${ }^{11}$ According to Giere, the basic representative relationship between models and the world is (pragmatic) similarity (Giere 2010, p. 269). That is to say, in virtue of certain specified similarities between the model and its target scientists represent certain aspects of the world (Giere 2004, pp. 747-748). What this means is that a model is considered to be similar to its target in certain respects and to certain degrees for a purpose $P$. Therefore, given the clarifier "in certain respects and to certain degrees," Giere equally suggests that idealization and abstraction can be accommodated within his pragmatic similarity approach. In the following two paragraphs, I shall pinpoint why the partial isomorphism account and the pragmatic similarity account fail to explicate scientific representation.

It is clear that da Costa and French, on the one hand, and, Giere, on the other hand, aim to explicate scientific representation. However, given the way that they seek to cash out scientific representation it is not at all clear that their respective accounts are able to single out scientific representations, for pragmatic similarity and partial isomorphism obtains in non-scientific representation as well. Consider Louis François Roubillac's famous statue of Isaac Newton at the Chapel of Trinity College, University of Cambridge. This statue is, as most would accept, clearly similar to the historical Isaac Newton. Although Roubillac added certain artistic elements for the purpose of providing a dignified representation of this particular Lucasian Professor of Mathematics, Newton's facial features are quite accurately represented. However, Roubillac's statue does not, for example, accurately represent Newton's true

${ }^{11}$ In what follows I shall focus on idealization and abstraction. An idealization refers to a conceptual scheme which deliberately distorts certain properties of a physical system when modelling it; an abstraction on the other hand refers to a conceptual scheme which omits certain properties of a physical system when modelling it (Cartwright 1989, pp. 185-198). 
stature and bodily proportions nor the true colours of his hair or skin. That is to say, Roubillac's statue is similar to Isaac Newton in certain respects and to certain degrees and in virtue of that it represents Newton. Based on similar considerations, one can equally maintain that partial isomorphism obtains between, for instance, the facial features of the statue and those of Isaac Newton and that it is in virtue of that that it represents Newton. This example can be accounted for by both accounts. The point here, however, is that the pragmatic similarity account and the partial isomorphism account do not have the resources to rule out the above example as a case of scientific representation. What this example suggests then is that, in order to account for scientific representation, something more is required than (1) introducing some kind of formal relation which obtains between a vehicle and its target, i.e. pragmatic similarity or partial isomorphism, and (2) focussing on the pragmatic and intentional considerations which enter into the ascription of such relations. Or, differently put, neither approach is explanatory of scientific representation. It is the search for that "something more" that, of course, motivates my own proposal on the matter. My claim will be that that "something else" enables us to single out scientific representations.

In a nutshell, my objection is that by explicating scientific representations in terms of certain formal relations, which obtain either "partially" or "in certain respects and to certain degrees," one cannot single out scientific representation proper. What needs to be incorporated in an account of scientific representation are the ways in which ascriptions of pragmatic similarity or partial isomorphism are arrived at. Such ascriptions are typically arrived at by the introduction of certain idealizing and abstracting assumptions. That is, the important thing to realize is that pragmatic similarity and partial isomorphism only obtain in view of certain idealizing and abstracting assumptions; or phrased differently, the idealizing and abstracting assumptions are that which ground users' ascriptions of pragmatic similarity or partial isomorphism between 
a vehicle and its target. ${ }^{12}$ As we will shortly see, in my account the introduction of certain idealizing and abstracting assumptions is what sets scientific representation apart from other forms of representation. The crucial difference between the account which will be spelled out in what follows, on the one hand, and the pragmatic similarity or partial isomorphism account, on the other, lies in the different way in which the relation between abstractionidealization and the existence of certain formal relations between the vehicle and its target is characterized. According to the latter, pragmatic similarity or partial isomorphism are the explanantes of idealization-abstraction; according to the former, it is the other way around: idealization and abstraction are the explanantes of the ascription of certain formal relations between the vehicle and its target. By placing idealization and abstraction at the heart of one's explication of scientific representation, as is done by introducing the notion of a pragmatic limiting case in $5.2,{ }^{13}$ the problem that vexes the pragmatic similarity account and the partial isomorphism account can be evaded, i.e. on the basis of this notion we will be able to single out scientific representations proper.

\subsection{Outline of the Limiting Case Account}

I shall begin by clarifying the notion of a pragmatic limiting case. To do so, I shall introduce the following conventions:

$$
\begin{aligned}
& \mathbf{0}_{M}=\text { the set of relevant objects of } M \\
& \mathbf{O}_{T}=\text { the set of relevant objects of } T \\
& \mathbf{R}_{M}=\text { the set of relevant relations among } \mathbf{O}_{M}
\end{aligned}
$$

\footnotetext{
${ }^{12}$ In Pincock 2005, pp. 1253-1255 related concerns are raised against partial isomorphism.

${ }^{13}$ That a model is a pragmatic limiting case of its target means that: (1) $M$ provides a ceteris paribus and ceteris absentibus conceptualization of its target i.e. it treats its target in a highly abstracted and idealized way, as it cuts loose from the complexity of the empirical world and deliberately distorts it - and (2) $M$ allows for the inference of certain relations which are not inferable from the target itself, but which hold approximately for the target relative to a purpose $P$.
} 


$$
\mathbf{R}_{T}=\text { the set of relevant relations among } \mathbf{O}_{T}
$$

It is very important to remark that properties, as in the remainder of this section, are construed as 1-ary relations and that relations include functions. Therefore when I speak of relations in what follows I refer to properties of objects - construed as 1-ary relations, relations among objects and functions between objects. That $M$ is a pragmatic limiting case of $T$ is then characterized as follows:

$M$ is a pragmatic limiting case of $T$ for purpose $P$, if and only if:

1. $\mathbf{R}_{M}$ describes an ideal ceteris paribus and ceteris absentibus state of affairs,

2. given the ideal state of affairs $\mathbf{R}_{M}$, a relation $\mathbf{r}$ holds exactly (where $\mathbf{r}$ is the sort of a relation of which we can determine the extent to which it holds empirically),

3. $\mathbf{r}$ obtains approximately for $\mathbf{R}_{T}$ relative to a purpose $\mathbf{P}$,

4. given that $\mathbf{r}$ holds exactly in $\mathbf{R}_{M}$ and approximately in $\mathbf{R}_{T}$ relative to a purpose $\mathbf{P}$, a one-on-one correspondence is predicated between $\mathbf{0}_{M}$ and $\mathbf{0}_{T}$ and between $\mathbf{R}_{M}$ and $\mathbf{R}_{T}$.

Given the above characterization, I propose the following characterization of scientific representation:

$M$ represents $T$ scientifically, if and only if, $M$ is a pragmatic limiting case of $T$ for purpose $P$.

An example is in order here. For some purposes, one may represent the orbital motion of the moon around the earth by a Newtonian one-body system. A one-body system provides an highly abstract and idealized picture of the moon's motion around the sun: it does 
not take into account other forces (for instance, the gravitational force of the sun), moreover, since the centre of the earth is considered as an empty force centre it does not take into account the moon's reaction force, and, finally, it treats the moon's orbital motion as perfectly circular and uniform. Given this ideal state of affairs one can establish that Kepler's area law holds exactly note that it can be determined empirically whether attracted bodies describe areas proportional to their times. Put differently, a onebody system provides us with an ideal state of affairs under which Kepler's area law would hold exactly; however, due to the presence of disturbing forces Kepler's area law does not hold exactly in our solar system, but only approximately. Correspondingly, in this model, the body will be interpreted as the moon, the circular trajectory will be interpreted as the actual path of the moon, and the empty centre of force will be interpreted as the centre of the earth. Similarly, the body orbiting around the empty force centre will stand for the moon orbiting around the earth and the body being attracted by the empty centre of force will stand for the moon being attracted by the earth. In contrast to Contessa's account, my account explains how denotation gets there in the first place: namely, because a relation, which obtains exactly under $\mathbf{R}_{M}$, holds approximately under $\mathbf{R}_{T}$ relative to a purpose $\mathbf{P}$. (By treating scientific representations as limiting cases of their corresponding targets, we are likewise able to explain how it is that users consider a model similar, quasi isomorphic or homomorphic to its targets in the first place.)

As will be shown below, the above limiting case account does not necessarily require that the relations among the $M$-objects, which are limiting cases of the relations among the $T$-objects, are first-order. First-order relations have only objects as their arguments. Correspondingly, second-order relations have firstorder relations as their arguments. And so on, for all other higherorder relations. When a set of first-order $M$-relations is a pragmatic limiting case of a set of first-order $T$-relations, I shall denote this as $\mathbf{R}_{M}^{O 1}$ is a pragmatic limiting case of $\mathbf{R}_{T}^{O 1}$. Constraining oneself to $\mathbf{R}^{01}$ does not seem to do justice to the potential abstractness of 
scientific representations. A probability model of a set of first-order relations is an illustration of $\mathbf{R}^{O 2}$, where a set of second-order $M$ relations (the probability distribution of certain first-order $M$ relations) is a limiting case of a set of second-order $T$-relations (the probability distribution of the corresponding first-order $T$-relations). Probability predicates (and the like) can be applied to second-order properties, in which case $\mathbf{R}^{O 3}$ obtains - and so on, for all higherorder cases. The outcome of this is that $\mathbf{R}^{O x}$ can potentially be very complex and situated at various levels of abstractness.

Let me provide a more complicated example. Quantum systems interacting with environment can be represented by a changing density operator $\rho(t)$ (Percival 1999, pp. 44-51). ${ }^{14}$ When physicists unravel a density matrix, they want to spell out a specific example of an ensemble which is equivalent to the given density matrix. In the case of an ensemble of qubits (i.e. two-state quantum systems), represented by a density operator equal to half the identity matrix, the unravellings are given by: $\rho=1 / 2=1 / 2(|z+\rangle+$ $\mid z->$ ) (i.e. an ensemble that is an equal mixture of spin up and spin down in the $\mathrm{z}$-direction) and $\rho=1 / 2=1 / 2(|\mathrm{x}+\rangle+|\mathrm{x}-\rangle)$ ) (i.e. an ensemble that is an equal mixture of spin up and spin down in the $\mathrm{x}$-direction). While an ensemble of quantum states has a density operator that satisfies a unique deterministic differential equation, the master equation corresponds to many different stochastic equations, i.e. different unravellings, for its components states (Percival 1999, p. 47). Unravelling the master equations means deriving a stochastic master equation from the master equation. Without further additional information about the environment, there is no way to choose one unravelling over the other (Spiller 1994, p. 168). If we do have additional information about the environment, we can associate the individual stochastic quantum state trajectories with the behaviour of individual quantum systems in the ensemble. One way of doing this is provided by Quantum State Diffusion (QSD). In general, QSD produces localization of a

${ }^{14}$ Another way of representing it is as an ensemble of changing pure states $|\psi(t)\rangle$ with probability distribution $\operatorname{Pr}(\psi(t))$. 
quantum state as it evolves in time (Percival 1999; Spiller, 1994 and 2002). If we obtain additional information about the environment that QSD is the preferred unravelling (compared to "quantum jumps" or other stochastic equations), we can unravel the master equation. The trajectories predicted by QSD are what you would expect if one were to make a particular set of measurements. Now, in order to formulate QSD one needs only the Hamiltonian and the Lindblad operators. The former represent the (internal) deterministic dynamics due to the change of $\rho$; the latter represent the effect of the (external) stochastic dynamics due to interaction with the environment (Percival 1999, p. 45). In order to arrive at exact equations one has to introduce idealizing conditions. A common idealizing assumption is that there is no systemenvironment interaction so that the Lindblads can be ignored and only the Hamiltonians remain. ${ }^{15}$ One can then further add the idealizing condition that the future of a system is only determined by its present state - in this case a system has Markov-behaviour. For the actual unravelling, we introduce the additional idealizing condition that the hypothetical measurements do not affect the system. The trajectories predicted by QSD are what you would expect if one were to make a particular set of measurements. It is obvious that unravelling the master equation by means of QSD involves several idealizing and abstracting conditions which characterize an ideal state of affairs $\mathbf{R}_{M}$. Under $\mathbf{R}_{M}$ the trajectories that one would expect if one were to make certain measurements are derived. Note, however, that here $\mathbf{R}_{M}$ involves a higher-order limiting case: one does not derive trajectories simpliciter, but the expected trajectories if one were to make measurements.

My characterization of scientific representation is clearly sufficient for scientific representation. If we accept that all scientific representations posit ceteris paribus and ceteris absentibus conditions, then it is also necessary for scientific representation. Additionally, the limiting case account has the right

${ }^{15}$ In the opposite limit, one assumes that the system is only determined by its environment so that the Hamiltonians can be ignored and only the Lindblads remain. 
logical properties of representation: to wit, asymmetry, nonreflexivity and non-transitivity (Suárez 2003, pp. 232-233; Frigg, 2006, pp. 60-61): given my characterization of scientific representations as limiting cases, $M$ is a limiting case of $T$ but not the other way around, $M$ cannot be a limiting case of itself, and since it is impossible that $T$ is a limiting case of another $M$ and that $M$ is a limiting case of another $M$, transitivity is ruled out. ${ }^{16}$ Moreover, the limiting case approach is able to account for misrepresentation, which refers to the fact that scientific representations are approximate or inaccurate in certain respects and to certain degrees, for the fact that models are idealizations and abstractions of their real-world targets is explicitly incorporated in my characterization of scientific representation. The limiting case account of scientific representation indeed squares nicely with the view that scientific representations involve idealizations and abstractions. As is commonly understood, scientific models presuppose certain conditions of which are strictly speaking false (Cartwright 1983). For instance, although we know that the earth is not a perfect sphere, in many Newtonian models we treat the earth as if it is a sphere. Similarly, the simple pendulum model provides an abstract and idealized description which assumes a zero air resistance and a very small angle of swing. To take another example, by means of Hardy-Weinberg equilibrium allele and genotype frequencies in a non-evolving population can be determined. Hardy-Weinberg equilibrium assumes that there is completely random mating and that there is no genetic mutation, natural selection, genetic migration or gene flow - conditions which do not hold in actual populations.

The above account also explains scientific representations' capacity for surrogate reasoning. By cutting loose from the complexities of the real world, scientific representations stipulate

${ }^{16}$ The formal criteria for transitivity are: if $\mathrm{A} \rightarrow \mathrm{B}$ and $\mathrm{B} \rightarrow \mathrm{C}$ then $\mathrm{A} \rightarrow \mathrm{C}$. When the arrow denotes the relation 'is a limiting case of,' then we have: if Model $_{1} \rightarrow$ Target $_{1}$ and Target $1 \rightarrow$ Model $_{2}$ then Model $_{1} \rightarrow$ Model $_{2}$. However, as the semantics of the limiting case approach rules out that Target ${ }_{1} \rightarrow$ Model $_{2}$ and Model $_{1} \rightarrow$ Model $_{2}$, transitivity cannot obtain. 
an ideal state of affairs in which certain relations, which cannot be directly inferred from the messy empirical world, can be inferred in an exact way. Scientific representations deliberately abstract from and idealize features of the physical world. This means that not all the features of a system are included (in the case of abstraction) and some of its features are deliberately distorted (in the case of idealization). Under such ceteris paribus and ceteris absentibus conditions, inferences can be drawn and relations can be established in a cognitive context where we do not have to deal with the full complexity of the real world.

\subsection{The Normative Question of Scientific Representation}

Readers will have noticed that I have said nothing on the conditions under which $\mathbf{r}$ obtains approximately for $\mathbf{R}_{T}$ relative to a purpose $\mathbf{P}$. The reason for this is straightforward: the issue of whether $\mathbf{r}$ holds sufficiently approximately pertains to the normative question of scientific representation. The particular goals at hand determine the degree of approximation we require from a scientific representation.

If our goals are very demanding, then we will require a very high degree of approximation. If, on the other hand, our goals aim only at giving a rough sketch, we will be satisfied with a lower degree of approximation. For instance, if we only want a rough sketch of a pendulum's gravitational force - let us say for educational means - we will use a very simple model. The standard simple pendulum model can be a perfectly appropriate model in the context of an introductory course in physics. By using the simple pendulum model, we assume that the cord of length $l$ is mass-less and that there is no air resistance. The period $T$ is then given by the formula $T=2 \times \pi \times\left(\frac{l}{g}\right)^{1 / 2}=2 \times \frac{\pi}{\omega}$, where $\omega$ refers to the angular frequency. Suppose now that in another context our goals are very stringent - for instance, consider the case of engineers who want to produce a detailed picture of the pendulum as a physical system for a particular technological application. One way to arrive at a more 
'realistic' ${ }^{17}$ model would be to incorporate the mass distribution. In this case, the period could be determined by the formula: $T=2 \times \pi$ $\times\left(\frac{I}{m \times g \times h}\right)^{1 / 2}$, where $I$ is the inertia around the axis of rotation, $m$ the total mass, and $h$ the distance from the axis and the centre of mass. If still not sufficiently detailed, we could add further factors to our pendulum model. There is no end to this process: one cannot include all necessary correction factors in one model. ${ }^{18}$

Given their inherent goal-dependency, the question of whether scientific representations are successful or adequate depends on the specifics of the goal which we seek to establish. This entails that there can be no general theoretical answer to the normative question of scientific representation.

Nevertheless, although scientific models abstract from and idealize properties of the natural world, we can determine empirically whether the features not covered by our model are indeed irrelevant with respect to a particular purpose. For instance, in the example of the simple pendulum model we can establish whether air resistance will significantly invalidate the results derived from this model by means of experimental set-ups given a purpose at hand. Comparison of pendulums in vacuo and pendulums on earth indeed shows that the deviations resulting from air resistance are for some purposes practically negligible however, not necessarily for all purposes. Scientific representations are constructed by human minds, but good ones are tested in the empirical world. More precisely, their inferential consequences and the non-significance of the factors, which they idealize or abstract from, can be confronted with phenomena and ascertained to be acceptable or not, in view of a purpose at hand. It is this process

${ }^{17}$ The quotation marks indicate that my proposal is neutral with respect to the realism-instrumentalism debate. It is highly desirable that an account of the normative problem of scientific representation is independent from the realisminstrumentalism debate - and equally so for an account of the constitution problem of scientific representation: for both realists and instrumentalists models represent scientifically.

18 Adam Morton has nicely discussed this idea in the context of atmospheric models (Morton 1993, pp. 660-662). 
which truly filters out adequate scientific representations, relative to specific cognitive and practical purposes.

\section{Conclusion}

By now I have explicated the pragmatic higher-order limiting case account, which, as I have tried to argue, provides an adequate treatment of the constitution problem of scientific representation. I have also highlighted its benefits and I have also explained why it fares better than its competitors: the inferential account, the interpretational account, the pragmatic similarity account, and the partial isomorphism account. If correct, my account provides a more adequate way to explicate scientific representation - at least, the debate on whether it does can now begin.

\section{References}

Bailer-Jones, D. (2003). When Scientific Models Represent. International Studies in the Philosophy of Science 17(1), 59-74.

Callender, C. and Cohen, J. (2006). There is No Special Problem About Scientific Representation. Theoria 21(1), 67-85.

Cartwright, N. (1983). How the Laws of Physics Lie. Oxford: Clarendon Press.

- (1989). Nature's Capacities and Their Measurement. Oxford: Clarendon Press.

Contessa, G. (2007). Representation, Interpretation and Surrogate Reasoning. Philosophy of Science 74(1), 48-68.

da Costa, N. C. A., and S. French (2003). Science and Partial Truth: A Unitary Approach to Models and Scientific Reasoning. Oxford: Oxford University Press.

French, S. (2003). A Model-Theoretic Account of Representation (or I Don't know Much About Art ... But I know It Involves Isomorphism). Philosophy of Science 70(5), Proceedings of the 2002 Biennial Meeting of the Philosophy of Science Association, Part I, S1472-S1483.

Frigg, R. (2006). Scientific Representation and the Semantic View of Theories, Theoria 21(1), pp. 49-65.

Giere, R. N. (1988). Explaining Science: A Cognitive Approach. Chicago: University of Chicago Press.

- (1999). Science without Laws. Chicago: University of Chicago Press. 
- (2004). How Models Are Used To Represent Physical Reality. Philosophy of Science 71(5), Proceedings of the 2002 Biennial Meeting of the Philosophy of Science Association, Part II, S742-S752.

- (2010). An Agent-based Conception of Models and Scientific Representation. Synthese 172(2), 269-281.

Hughes, R. I. G. (1997). Models and Representation. Philosophy of Science 64(5), Proceedings of the Biennial Meeting of the Philosophy of Science Association, Part II, S325-S336.

Morton, A. (1993). Mathematical Models: Questions of Trustworthiness. The British Journal for the Philosophy of Science 44(4), 659-674.

Mundy, B. (1986). On the General Theory of Meaningful Representation. Synthese 67(3), 391-437.

Percival, I. C. (1999). Quantum State Diffusion. Cambridge: Cambridge University Press.

Pincock, C (2005). Overextending Partial Structures: Idealization and Abstraction, Philosophy of Science 72(5), Proceedings of the 2004 Biennial Meeting of the Philosophy of Science Association, Part I, 12481259.

Suárez, M. (2003). Scientific Representation: Against Similarity and Isomorphism. International Studies in the Philosophy of Science 17(3), 225-244.

- (2004). An Inferential Conception of Scientific Representation. Philosophy of Science 71(5), Proceedings of the 2002 Biennial Meeting of the Philosophy of Science Association, Part II, S767-S779.

Spiller, T. P. (1994). The Zeno Effect: Measurement versus Decoherence. Physics Letters A 192, 163-168.

- (2002). Review of Ian C. Percival's Quantum State Diffusion. Studies in History and Philosophy of Modern Physics 33B(4), 707-716.

Teller, P. (2001). Twilight of the Perfect Model Model. Erkenntnis 55(3), 393-415.

van Fraassen, B. C. (1980). The Scientific Image. Oxford: Clarendon Press.

- (1994). Interpretation of Science; Science as Interpretation. In J. Hilgevoord (Ed.), Physics and Our View of the World. Cambridge: Cambridge University Press.

- (2008). Scientific Representation: Paradoxes of Perspective. Oxford: Clarendon Press. 